\title{
Fear Extinction across Development: The Involvement of the Medial Prefrontal Cortex as Assessed by Temporary Inactivation and Immunohistochemistry
}

\author{
Jee Hyun Kim, ${ }^{1}$ Adam S. Hamlin, ${ }^{2}$ and Rick Richardson ${ }^{1}$ \\ ${ }^{1}$ School of Psychology, The University of New South Wales, Kensington, Sydney, New South Wales 2052, Australia, and ${ }^{2}$ Queensland Brain Institute, \\ University of Queensland, St Lucia, Queensland 4072, Australia
}

\begin{abstract}
Extinction in adult animals, including humans, appears to involve the medial prefrontal cortex (mPFC). However, the role of mPFC in extinction across development has not yet been studied. Given several recent demonstrations of developmental differences in extinction of conditioned fear at a behavioral level, different neural circuitries may mediate fear extinction across development. In all experiments, noise conditioned stimulus (CS) and shock unconditioned stimulus (US) were used. In experiment 1A, temporary unilateral inactivation of the mPFC during extinction training impaired long-term extinction the following day in postnatal day 24 (P24) rats but not in P17 rats. In experiment 1B, bilateral inactivation of the MPFC again failed to disrupt long-term extinction in P17 rats. In experiment 2, extinction training increased phosphorylated mitogenactivated protein kinase (pMAPK) in the MPFC for P24 rats but not for P17 rats, whereas rats of both ages displayed elevated pMAPK in the amygdala. Across both ages, "not trained," "reactivated," and "no extinction" control groups expressed very low numbers of pMAPKimmunoreactive (IR) neurons across both neural structures. This result indicates that the mere conditioning experience, the exposure to the CS, or the expression of CS-elicited fear in and of itself is not sufficient to explain the observed increase in PMAPK-IR neurons in the mPFC and/or the amygdala after extinction. Together, these findings show that extinction in P17 rats does not involve the mPFC, which has important theoretical and clinical implications for the treatment of anxiety disorders in humans.
\end{abstract}

\section{Introduction}

It is a long-held belief that early experiences have a profound impact on later behavior. In particular, several theories suggest that a traumatic event early in life forms the basis of adult anxiety disorders (Jacobs and Nadel, 1999; Mineka and Zinbarg, 2006). Further, anxiety disorders emerge quite early in life and individuals first diagnosed with an anxiety disorder in childhood express more severe symptoms than do individuals first diagnosed in adulthood (Newman et al., 1996). It is surprising then that not much is known about the treatment of anxiety disorders in children and adolescents (Pine et al., 2009).

The most used treatment for anxiety disorders is some type of exposure therapy that relies on the process of extinction (Davis and Myers, 2002). Extinction is the decrease in conditioned response to a previously trained conditioned stimulus (CS) after nonreinforced presentations of the CS (Pavlov, 1927). Despite suggestions that fear acquired early in development is particularly resistant to extinction (e.g., Jacobs and Nadel, 1999), recent studies report not only that fear is extinguished but also that it may even be erased early in development (Kim and Richardson, 2008). For example, adult rats typically show a relapse in extin-

Received Feb. 4, 2009; revised June 24, 2009; accepted July 14, 2009.

This research was supported by an Australian Postgraduate Award (J.H.K.) and Discovery Grants DP0346139 and DP0666953 (R.R.) from the Australian Research Council.

Correspondence should be addressed to Dr. Jee Hyun Kim, School of Psychology, The University of New South Wales, Sydney, NSW 2052, Australia. E-mail: jkim@psy.unsw.edu.au.

DOI:10.1523/JNEUROSCI.0596-09.2009

Copyright $\odot 2009$ Society for Neuroscience $\quad$ 0270-6474/09/2910802-07\$15.00/0 guished fear (e.g., renewal and reinstatement). However, preweanling-aged rats [i.e., postnatal day 17 (P17)] do not exhibit renewal or reinstatement, whereas postweanling-aged rats (e.g., P24) do (Kim and Richardson, 2007a,b). Also, systemic injection of the GABA receptor inverse agonist FG7142 reverses extinction in adult rats and P24 rats but not in P17 rats (Harris and Westbrook, 1998; Kim and Richardson, 2007a).

These studies on fear extinction across development strongly suggest that once extinguished, fear does not return in P17 rats. It appears that qualitatively different neural mechanisms underlie extinction across development. However, virtually nothing is known about the neural circuitry subserving fear extinction in developing rats. It is well documented that the MPFC is critical for extinction retention in adult rats (for review, see Quirk and Mueller, 2008). For example, permanent and temporary lesions of the ventral mPFC impair long-term extinction (e.g., Morgan et al., 2003; Sierra-Mercado et al., 2006), and neural activity in this structure predicts the strength of extinction expression (Milad and Quirk, 2002). Additionally, extinction is disrupted by blockade of mitogen-activated protein kinase/extracellular signal-regulated kinase (MAPK/ERK) signaling pathway in the mPFC (Hugues et al., 2004, 2006); this pathway is a part of the intracellular cascade that is involved in producing long-lasting changes in synaptic efficacy (Kandel, 2001). Despite the importance of the mPFC in extinction in adult rats, however, no one has yet examined whether the mPFC is also necessary for extinction in younger rats.

In experiments $1 \mathrm{~A}$ and $1 \mathrm{~B}$ we show that temporary inactivation of the mPFC during extinction training impaired long-term 
extinction in P24 rats but not in P17 rats. In a follow-up experiment, we observed increased numbers of phosphorylated MAPK-immunoreactive (pMAPK-IR) neurons in the mPFC and amygdala in P24 rats after extinction training. However, an elevated number of pMAPK-IR neurons was observed only in the amygdala in P17 rats after extinction training.

\section{Materials and Methods}

Subjects and surgery

Male Sprague Dawley-derived rats were bred and housed in the School of Psychology, University of New South Wales, as described in previous studies (Kim and Richardson, 2007a,b). No more than one rat per litter was used per group. Group sizes were six to nine in experiments $1 \mathrm{~A}$ and $1 \mathrm{~B}$ and four to seven in experiment 2. All surgical procedures were similar to those previously described (Kim and Richardson, 2008). One day before training, rats were anesthetized with isoflurane (Laser Animal Health) mixed with oxygen and nitrous oxide. A 26-gauge guide cannula (Bioscientific) was unilaterally implanted in the mPFC in experiment 1A and identical double cannula (center-to-center distance, $1.2 \mathrm{~mm}$ ) was bilaterally implanted in the mPFC in experiment $1 \mathrm{~B}$. The stereotaxic coordinates were $2.7 \mathrm{~mm}$ anterior, $0.6 \mathrm{~mm}$ lateral/bilateral, $3.8 \mathrm{~mm}$ ventral to bregma. After surgery, rats were injected subcutaneously with 0.03 $\mathrm{ml}$ each of a $100 \mathrm{mg} / \mathrm{ml}$ solution of cephazolin and a $300 \mathrm{mg} / \mathrm{ml}$ solution of benacillin. It has previously been shown that rats these ages recover from surgery well within $24 \mathrm{~h}$ (Weber and Richardson, 2001; Kim and Richardson, 2008). Rats were decapitated $<1 \mathrm{~h}$ after the final test, and tissue sections were collected and stained for Nissl substance for verification of cannula placement. In experiment 1A, data of two rats from the P24-saline group and one rat from the P24-vehicle group were removed from statistical analyses because of lesions of the mPFC. All other cannula placements were within the mPFC. In experiment $1 \mathrm{~B}$, data of one rat from the saline group and three rats from the muscimol group were removed from statistical analyses because of lesions of the mPFC. All other cannula placements were within the mPFC.

\section{Drug infusion}

Five minutes before extinction training, the cannula stylets were removed and 33-gauge injection cannulas, extending $1 \mathrm{~mm}$ below the tip of the guide cannula, were inserted for infusion of muscimol (Sigma-Aldrich) or saline. Muscimol was dissolved in $0.9 \%$ (weight/volume) sterile physiological saline and given in a dose of $0.6 \mu \mathrm{g}$ in $0.5 \mu \mathrm{l}$ infused over 2 min using a microsyringe driven by a microinfusion pump (syringe pump SP101IZ, World Precision Instruments) via PE50 tubing. The injection cannulas were left in position for an additional 2 min before withdrawal.

\section{Apparatus and procedures}

Two types of experimental chambers were used to provide different contexts and they were the same as those previously described (Kim and Richardson, 2007a,b). One type was rectangular $(13.5 \mathrm{~cm}$ long $\times 9 \mathrm{~cm}$ wide $\times 9 \mathrm{~cm}$ high), with the front wall, rear wall, and ceiling constructed of clear Plexiglas (the two side walls consisted of $3 \mathrm{~mm}$ stainless steel rods set $1 \mathrm{~cm}$ apart). The second type was rectangular $(30 \mathrm{~cm}$ long $\times 30 \mathrm{~cm}$ wide $\times 23 \mathrm{~cm}$ high) and wholly constructed of Plexiglas with two side walls that consisted of vertical black and white stripes. The floor of both chambers consisted of $3 \mathrm{~mm}$ stainless steel rods set $1 \mathrm{~cm}$ apart connected to a custom built constant-current shock generator. All training occurred in the first type, and all extinction and test sessions occurred in the second type of chamber. The CS was a white noise ( $8 \mathrm{~dB}$ above background).

Training. After a 2-min adaptation period, the CS was presented for $10 \mathrm{~s}$. The shock unconditioned stimulus (US) $(0.6 \mathrm{~mA}, 1 \mathrm{~s})$ was administered during the last second of the CS. P17 rats received six pairings and P24 rats received three pairings of the CS and US to equate the initial levels of learning. The intertrial interval (ITI) ranged from $85 \mathrm{~s}$ to $135 \mathrm{~s}$ with a mean of $110 \mathrm{~s}$.

Extinction. The extinction session consisted of the presentation of five blocks of 6 CS presentations in the absence of shock (total of 30 CS presentations). After a 2-min adaptation period, the $10 \mathrm{~s}$ CS was presented with a 10 s ITI.
Testing. The baseline level of freezing in the absence of the CS was scored for $1 \mathrm{~min}$. The CS was then presented for $2 \mathrm{~min}$.

Freezing. Freezing is defined as the absence of all movement other than that required for respiration (Fanselow, 1980). Freezing was scored by a time sampling procedure whereby each rat was scored every $3 \mathrm{~s}$ as freezing or not freezing by a blind observer. A second-scorer unaware of the experimental conditions scored $70 \%$ of rats across all experiments. The inter-rater reliability was very high $(r=0.95)$.

Tissue processing, immunohistochemistry, and neuronal counting

Tissue processing. One hour after extinction in experiment 2, rats were deeply anesthetized with sodium pentobarbital (100 mg/kg, i.p.) and transcardially perfused with $50 \mathrm{ml}$ of $0.9 \%$ saline, containing $1 \%$ sodium nitrite and heparin (5000 i.u./ml), followed by $150 \mathrm{ml}$ of $4 \%$ paraformaldehyde in $0.1 \mathrm{~m}$ phosphate buffer (PB), pH 7.4. Brains were postfixed for $1 \mathrm{~h}$ in the same fixative and placed in $20 \%$ sucrose solution overnight. Brains were blocked using a matrix (Stoelting) aligned to the rat brain atlas (Paxinos and Watson, 1998), and $40 \mu \mathrm{m}$ coronal sections were cut using a cryostat (Microm HM 560) in four serially adjacent sets and stored in $0.1 \%$ sodium azide in $0.1 \mathrm{M} \mathrm{PB}$ saline ( $\mathrm{pH} 7.2$ ).

Immunohistochemistry. Series of sections were selected from each rat and immunohistochemistry was used to reveal phospho-p44-42MAPK. Free-floating sections were washed repeatedly in $0.1 \mathrm{M} \mathrm{PB} \mathrm{(pH} \mathrm{7.4)} \mathrm{fol-}$ lowed by two 30 min washes in $50 \%$ ethanol, the second of which contained $3 \% \mathrm{H}_{2} \mathrm{O}_{2}$, and were then incubated in 5\% normal horse serum (NHS) in PB (pH 7.4) for $30 \mathrm{~min}$. Sections were then incubated in rabbit antiserum against pMAPK [1:500; phospho-p44-42MAPK (Thr202/ Tyr204), Cell Signaling Technology] for $48 \mathrm{~h}$ at $4^{\circ} \mathrm{C}$, with gentle agitation. The primary antibody was diluted in PBT-X, which consisted of 0.1 м PB (pH 7.4) containing $2 \%$ NHS and $0.2 \%$ Triton X-100. After washing off unbound primary antibody, sections were incubated overnight at room temperature in biotinylated donkey anti-rabbit IgG (1: 1,000; Jackson ImmunoResearch Laboratories) diluted in PBT-X. After being washed, sections were then incubated for $2 \mathrm{~h}$ at room temperature in $\mathrm{ABC}$ reagent $(6 \mu \mathrm{l} / \mathrm{ml}$ avidin and $6 \mu \mathrm{l} / \mathrm{ml}$ biotin; Vector Elite kit; Vector Laboratories). Black immunoreactive cytoplasm labeled for pMAPK was revealed by a nickel-intensified diaminobenzidine (DAB) reaction, with peroxide being generated by glucose oxidase. In this DAB reaction, sections were washed in $\mathrm{PB}(\mathrm{pH} 7.4)$ followed by $0.1 \mathrm{M}$ acetate buffer ( $\mathrm{pH}$ 6.0) and then incubated for $15 \mathrm{~min}$ in $0.1 \mathrm{M}$ acetate buffer $(\mathrm{pH}$ 6.0 ) containing $2 \%$ nickel sulfate, $0.025 \% 3,3-\mathrm{DAB}, 0.04 \%$ ammonium chloride, and $0.02 \% \mathrm{D}$-glucose. The peroxidase reaction was started by adding $0.2 \mu \mathrm{l} / \mathrm{ml}$ glucose oxidase and stopped using acetate buffer $(\mathrm{pH}$ 6.0). Brain sections were then washed in PB (pH 7.4). Sections were mounted onto gelatin-treated slides, dehydrated, cleared in xylene, and coverslipped with DPX (Biolab).

Neuronal counting. The quantification of phospho-MAPK immunoreactivity was performed at $100 \times$ magnification using manual blind counts of neurons. All sections counted were $160 \mu \mathrm{m}$ apart. The brain regions analyzed were as follows: $\mathrm{mPFC}$ over three sections $(3.20 \mathrm{~mm}-$ $2.88 \mathrm{~mm}$ anterior to bregma) and bed nucleus of the stria terminalis intra-amygdaloid division (BSTIA) and basolateral amygdala (BLA) over six sections $(2.20 \mathrm{~mm}-3.10 \mathrm{~mm}$ posterior to bregma). In each rat, pMAPK-IR was quantified within predefined boundaries delineating each neural structure according to the rat brain atlas of Paxinos and Watson (1998). The top border of the prelimbic cortex (PrL) was identified by aligning the counting grid with the top border of the corpus callosum (2.5 $\mathrm{mm}$ ventral to bregma).

\section{Results}

\section{Experiments 1A and 1B: the $\mathrm{MPFC}$ is critical for extinction in $\mathrm{P} 24$ rats but not in $\mathrm{P} 17$ rats}

To test the involvement of the mPFC in fear extinction across development, in experiment 1A we trained P17 and P24 rats on a Pavlovian fear conditioning procedure in which we gave pairings of a white noise CS and a footshock US. One day later, all rats received CS-alone presentations (i.e., extinction). Before extinction training, rats were microinfused with either saline or the 
GABAergic agonist muscimol into the mPFC (Fig. 1A shows the cannula tip placements). During extinction training, all groups exhibited substantial levels of CS-elicited freezing in the first block that decreased by the last block (mixed-design ANOVA; $\left.F_{(4,100)}=105.77 ; p<0.0001\right)$ (Fig. $1 B$, left). There were no significant differences across age or drug condition, nor any significant interactions.

At testing the next day (drug free), there were no differences in baseline levels of freezing (Table 1). More importantly, P24 rats that received a preextinction microinfusion of muscimol exhibited substantially higher levels of CS-elicited freezing compared with P24 rats that had received saline, whereas P17 rats showed very low levels of freezing (i.e., good extinction retention) regardless of drug condition (Fig. $1 B$, right). ANOVA revealed significant effects of age $\left(F_{(1,25)}=4.70 ; p<0.05\right)$, drug $\left(F_{(1,25)}=4.43 ; p<\right.$ $0.05)$, and an age $\times$ drug interaction $\left(F_{(1,25)}=5.43 ; p<0.05\right)$. To understand the significant age $\times$ drug interaction, we performed $t$ tests at each age. These analyses revealed that microinfusion of muscimol blocked long-term extinction in P24 rats $\left(t_{(11)}=3.63 ; p<0.005\right)$ but had no effect in P17 rats $(t<1)$. These results show that the mPFC is not necessary for extinction of conditioned fear in $\mathrm{P} 17$ rats.

In experiment $1 \mathrm{~B}$, we trained $\mathrm{P} 17$ rats as described in experiment 1A. One day later, two groups of rats received CS-alone presentations (i.e., extinction), whereas another group was exposed to the context for the equal amount of time without any CS presentations (the no-extinction group). Before extinction training, rats were microinfused with either saline or the GABAergic agonist muscimol into the mPFC. The no-extinction group received either saline or muscimol before the context exposure. Figure $2 A$ shows the cannula tip placements. The two extinction groups exhibited substantial levels of CS-elicited freezing in the first block that decreased by the last block $\left(F_{(4,52)}=13.93 ; p<\right.$ 0.0001 ) (Fig. $2 B$, left). There were no significant differences across drug condition, nor any significant interactions.

At testing the next day (drug free), there were no differences in baseline levels of freezing (Table 1). There were also no differences between rats that were infused with saline or muscimol in the no-extinction group $(p=0.58)$; therefore, the data were collapsed into a single no-extinction group. Bilateral inactivation of the mPFC had no effects on long-term extinction in P17 rats (Fig. $2 \mathrm{~B}$, right). One-way ANOVA indicated that there were group differences in CS-elicited freezing at testing $\left(F_{(2,21)}=8.78\right.$; $p<0.005$ ), and post hoc comparisons [Tukey's Honestly Significant Difference (HSD)] showed that the no-extinction group displayed significantly higher CS-elicited freezing at testing compared with either of the extinction groups ( $p$ values $<0.05$ ). This result replicates the findings of experiment $1 \mathrm{~A}$ and shows that the MPFC is not critical for fear extinction in P17 rats.

\section{Experiment 2: P24 but not P17 rats show elevated pMAPK in the $\mathrm{mPFC}$ after extinction}

To gain a further insight into the neural circuitry underlying fear extinction across development, experiment 2 examined activation of the MPFC and the amygdala by quantifying the phosphorylation of MAPK in these structures after extinction training. It has been shown in adult rats that extinction led to increased
Table 1. Mean levels of baseline freezing at test in experiments $1 A$ and 1B

\begin{tabular}{llc}
\hline Experiment & Group & Mean \% freezing at baseline ( \pm SEM) \\
\hline 1A & P17-saline & $7.5( \pm 2)$ \\
& P17-muscimol & $9.4( \pm 6)$ \\
& P24-saline & $24.2( \pm 9)$ \\
1B & P24-muscimol & $10.7( \pm 5)$ \\
& No extinction & $13.9( \pm 4)$ \\
& Saline & $11.9( \pm 3)$ \\
& Muscimol & $12.1( \pm 2)$ \\
\hline
\end{tabular}

No significant differences were found between any groups.

MAPK/ERK in the BLA (Herry et al., 2006). In the present experiment, P17 and P24 rats were trained with CS-US pairings or received CS presentations without the US (groups not trained). One day later, trained rats received either five blocks of CS-alone presentations as described in experiments $1 \mathrm{~A}$ and $1 \mathrm{~B}$ (extinction groups), context exposure only (no-extinction groups), or one block of CS-alone presentation (reactivated groups). Groups not trained received equivalent CS-alone presentations as the extinction groups. All rats were perfused $1 \mathrm{~h}$ after the end of the extinction, reactivation, or context exposure session.

Extinction training proceeded uneventfully (Fig. $3 A$ ). CSelicited freezing data for the first block were initially analyzed separately to assess whether groups reactivated displayed equal levels of fear as extinction groups. ANOVA with post hoc pairwise comparisons (Tukey's HSD) revealed that reactivated and extinction groups did not differ $(p=0.76)$ and both groups froze significantly more than groups not trained $\left(F_{(2,25)}=58.68\right.$; $p<$ $0.0001)$. There was no effect of age or interaction of age and group ( $p$ values $>0.8$ ). Subsequent mixed-design ANOVA of extinction data included extinction and untrained groups. There were significant effects of block $\left(F_{(4,76)}=8.69 ; p<0.0001\right)$ and group $\left(F_{(1,19)}=76.85 ; p<0.0001\right)$ and a significant block $\times$ group interaction $\left(F_{(4,76)}=9.8 ; p<0.0001\right)$. There were no significant differences across age, nor any other significant interactions. These analyses indicate that P24 and P17 rats that received training showed significant within-session extinction whereas rats not trained showed negligible CS-elicited freezing.

Figure $3 B$, left and right, presents the number of pMAPK-IR neurons observed in the prelimbic and infralimbic cortices of the mPFC, respectively. Extinction of conditioned fear led to an elevated phosphorylation of MAPK in the MPFC in P24 rats; in contrast, P17 rats showed negligible numbers of pMAPK-IR neurons in the $\mathrm{mPFC}$ across all conditions. Analysis of the data from the PrL revealed a significant effect of age $\left(F_{(1,34)}=24.52 ; p<\right.$ 

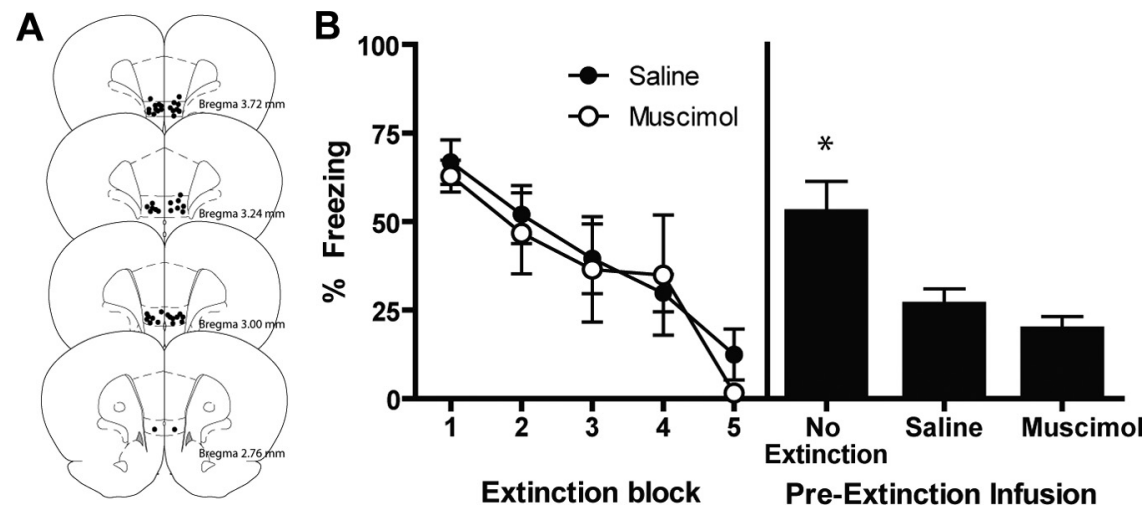

Figure 2. The effect of reversible bilateral inactivation of the MPFC in extinction in the developing rat. P17 (O). $\boldsymbol{A}$, Histological reconstruction of coronal sections showing cannula placements in the mPFC. $\boldsymbol{B}$, Mean ( \pm SEM) CS-elicited freezing at extinction and test. Bilateral inactivation of the mPFC during extinction had no effects on long-term extinction in P17 rats. *Significant difference $(p<0.05)$ from the other groups.
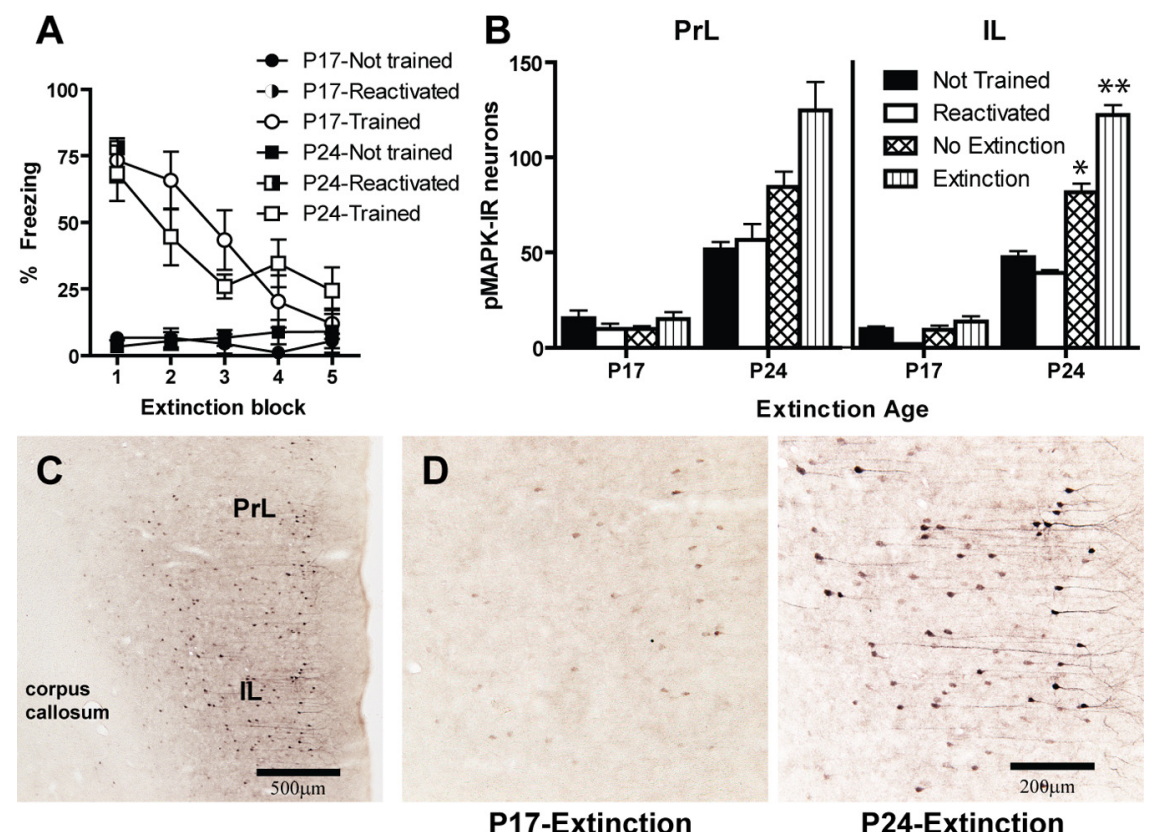

P17-Extinction

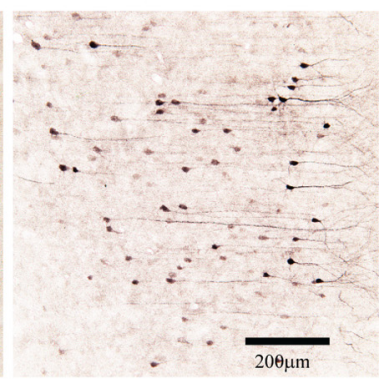

P24-Extinction

Figure 3. $A, \operatorname{Mean}( \pm$ SEM) CS-elicited freezing at extinction. Reactivated rats showed amounts of CS-elicited freezing equal to those of extinction rats during the first block of extinction. Only rats that were trained and extinguished showed significant extinction across trial; rats not trained did not show any CS-elicited freezing. $\boldsymbol{B}$, Numbers of pMAPK-IR neurons in the PrL and IL after extinction training in P17 and P24 rats. Extinction of conditioned fear led to an elevated number of pMAPK-IR neurons in IL in P24 rats but not in P17 rats. *Significant difference $(p<0.005)$ from not trained and reactivated groups of the same age. ${ }^{*}$ Significant difference $(p<0.005)$ from all other groups of the same age. $\boldsymbol{C}, \boldsymbol{D}$, Photomicrograph of pMAPK-IR neuronal staining in the medial prefrontal cortex ( $(\boldsymbol{C})$ and pMAPK-IR neuronal staining in the IL in the trained- extinction group from a P17 rat (left) and a P24 rat (right) (D).

$0.0001)$, but the effect of group and age $\times$ group interaction failed to reach significance ( $p$ values $>0.16$ ). Despite the nonsignificant interaction, post hoc Tukey's HSD comparisons were performed for P24 rats because the means appear to be quite different. Nevertheless, there were no significant differences between any groups ( $p$ values $>0.17$ ). Analysis of the data from the infralimbic cortex (IL) revealed significant effects of age $\left(F_{(1,34)}=133.34 ; p<0.0001\right)$, group $\left(F_{(3,34)}=15.28 ; p<\right.$ $0.0001)$, and an age $\times$ group interaction $\left(F_{(3,34)}=10.09 ; p<\right.$ $0.0001)$. To understand the significant age $\times$ group interaction, post hoc comparisons (Tukey's HSD) were done at each age. In P24 rats, the extinction group expressed a significantly higher number of pMAPK-IR neurons in the IL compared with the other groups
( $p$ values $<0.05)$. Also, the no-extinction group displayed a significantly higher number of pMAPK-IR neurons than the reactivated group $(p<0.05)$. This result may have been caused by the rats in the noextinction group having some generalized freezing that may have been extinguished as a result of the prolonged exposure to the novel context; however, no conclusions can be drawn from the present study. In $\mathrm{P} 17$ rats, no differences were found among any groups ( $p$ values $>0.23)$. Together, these data show that extinction training in P24 leads to phosphorylation of MAPK in the MPFC, especially in the IL. However, extinction has no effects on the number of pMAPK-IR neurons in the mPFC in P17 rats. These results are consistent with experiment 1, where it was shown that reversible inactivation of the mPFC impaired long-term extinction in P24 rats but not in P17 rats. Photomicrographs of the immunostained neurons in the mPFC are shown in Figure $3 C, D$.

In contrast to the developmental differences observed in the mPFC, extinction led to increased numbers of pMAPK-IR neurons in the amygdala in both P17 and P24 rats. Specifically, rats in the extinction condition at both ages showed significantly higher phosphorylation of MAPK in the BLA (Fig. 4A) and in the BSTIA (Fig. $4 B$ ). In the BLA, there were significant effects of group $\left(F_{(3,34)}=4.29 ; p<\right.$ $0.05)$ and age $\left(F_{(1,34)}=5.12 ; p<0.05\right)$ but no age $\times$ group interaction $(p=0.66)$. It appears that P24 rats generally express more pMAPK-IR neurons in the BLA across different conditions, as indicated by the main effect of age. Post hoc Tukey's HSD comparisons showed that extinction groups had significantly higher numbers of pMAPK-IR neurons than other groups across the ages. In the BSTIA, there was a significant effect of group $\left(F_{(3,34)}=5.75\right.$; $p<0.005)$ but no effect of age or age $\times$ group interaction ( $p$ values $>0.99$ ); post hoc comparisons (Tukey's HSD) showed that extinction groups had significantly higher numbers of pMAPK-IR neurons than other groups in both P24 and P17 rats. These results indicate that extinction activates the BLA and the BSTIA in P17 and P24 rats. The present finding that extinction leads to an increase in activity in the amygdala across development is consistent with a previous study reporting that temporary inactivation of the amygdala impairs extinction in both P17 and P24 rats (Kim and Richardson, 2008). Photomicrographs of the immunostained neurons in the amygdala are shown in Figure 4C-E.

We also examined whether there were age-related differences in the correlations between the PrL, IL, BLA, and BSTIA in the phosphorylation of MAPK after extinction (Table 2). Of particular interest was the correlation between activity in the amygdala 
subnuclei and the mPFC subnuclei. In adult rats, the amygdala and MPFC are densely interconnected (Sesack et al., 1989; Condé et al., 1995) and extinction of fear in adulthood appears to critically depend on these connections (Milad and Quirk, 2002; Hobin et al., 2003). Therefore, activity in the amygdala and the mPFC as measured by pMAPK-IR neurons may correlate as a result of extinction training in P24 rats. In P17 rats, however, we may observe a different pattern of correlation. Statistical analyses in P24 rats with Pearson's correlation coefficient showed that phosphorylation of MAPK in the BLA significantly correlated with those in the IL $(r=0.45)$ but not with those in the BSTIA $(r=-0.08)$ or the PrL $(r=0.14)$. Also, pMAPK-IR neuron counts in the BSTIA significantly correlated with those in the $\operatorname{PrL}(r=0.68)$ and the IL $(r=0.58)$. The PrL and IL also showed correlated activity with each other $(r=0.67)$. In P17 rats, however, only the PrL and the IL showed significant correlated phosphorylation of MAPK $(r=0.56)$. No other significant correlations were found at this age. These results suggest that by $24 \mathrm{~d}$ of age the mPFC is working in conjunction with the amygdala. On the other hand, the $\mathrm{mPFC}$ and the amygdala may not to be working together during extinction of fear in $\mathrm{P} 17$ rats.

\section{Discussion}

Here we show that temporary inactivation of the MPFC at the time of extinction training blocks extinction retention the following day in P24 rats but not in P17 rats. Further, immunohistochemical analyses revealed that extinction of conditioned fear involves intracellular activation of the IL and the amygdala in P24 rats, whereas only the amygdala is activated in P17 rats. It is interesting that the BSTIA of the amygdala showed a significant increase in numbers of pMAPK-IR neurons after extinction, because the GABAergic intercalated cells that are strongly implicated in extinction (Likhtik et al., 2008) have been shown to be located mainly in the BSTIA (McDonald, 1985; Millhouse, 1986), although it cannot be verified that the neurostaining observed in the BSTIA in the present study includes the intercalated cells. It should also be noted that the increased number of pMAPK-IR neurons observed in the extinction groups was over and above that of untrained, reactivated, and no-extinction groups. That is, the increase in phosphorylation of MAPK was specifically because of extinction training rather than the mere experience of being exposed to $30 \mathrm{CS}$ presentations, expressing fear, or being conditioned. Together, the present experiments show that extinction at $17 \mathrm{~d}$ of age does not involve the mPFC, suggesting that the neural mechanisms underlying extinction at this age are fundamentally different from those in older rats.

Theoretically, it is widely accepted that extinction training in adult rats leads to a CS-no-US association that coexists with the original CS-US association learned during conditioning (e.g., Bouton, 2002). This dual memory of the CS then allows the rat to
BLA

B

BSTIA

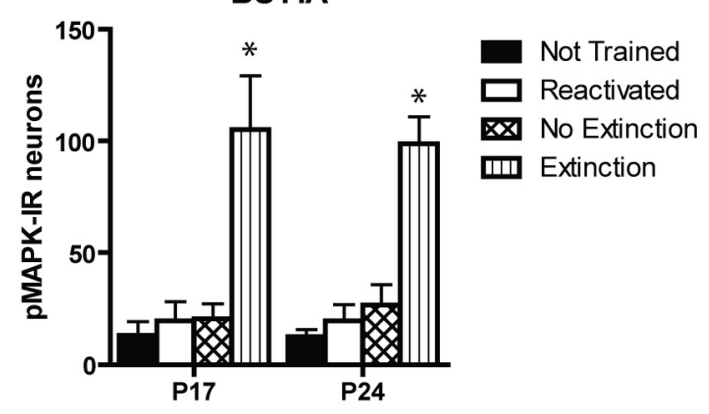

Extinction Age

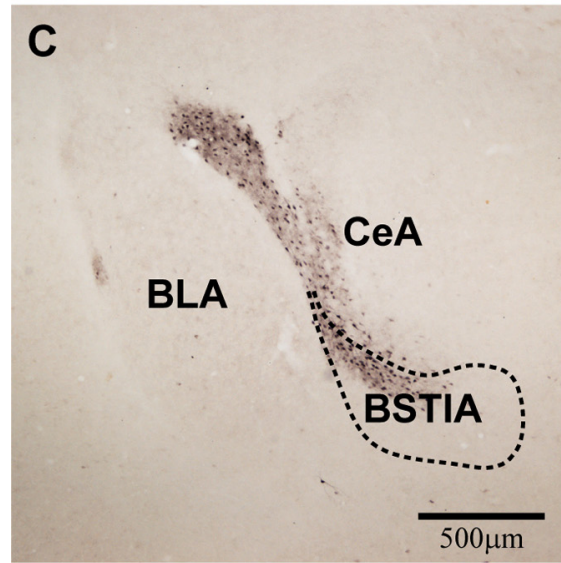

D

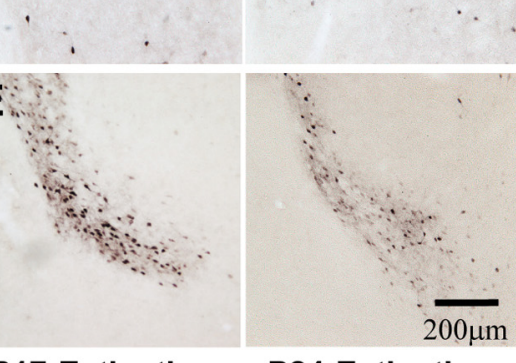

P17-Extinction

Figure 4. $\boldsymbol{A}$, Numbers of pMAPK-IR neurons in the BLA after extinction training in P24 and P17 rats. *Significant effect of extion across both ages $(p<0.05)$. $\boldsymbol{B}$, Numbers of pMAPK-IR neurons in the BSTIA after extinction training in P24 and P17 rats. neurons in the BLA and BSTIA at both ages. C, Photomicrograph of pMAPK-IR neuronal staining in the amygdala. D, E, pMAPK-IR neuronal staining in the BLA in the trained-extinction group from a P17 rat (left) and a P24 rat (right) (D) and pMAPK-IR neuronal staining in the BSTIA in trained- extinction group from a P17 rat (left) and a P24 rat (right) (E). CeA, Central amygdala.

Table 2. Correlations between PrL, IL, BLA, and BSTIA in pMAPK expression in P24 and $\mathrm{P} 17$ rats

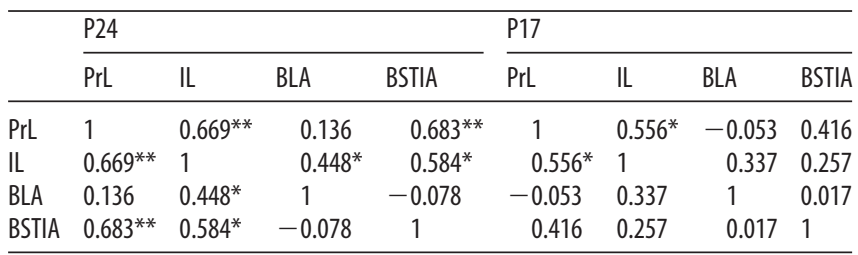

*Correlation is significant at the 0.05 level (two tailed); ${ }^{* *}$ correlation is significant at the 0.01 level (two tailed).

express the appropriate response according to the contextual information provided at the time of test. This flexibility in the expression of memory is possible because the formation and the storage of extinction learning do not depend on a single neural structure but rather depend on a network of several neural structures. Specifically, current dominant models propose that extinction is expressed when the mPFC activates the GABAergic intercalated neurons, which then inhibit the output of the amygdala. However, in a context that is different from where extinction had occurred, the hippocampus inhibits the mPFC, thereby releasing the amygdala from inhibition, which results in the expression of fear (Hobin et al., 2003; Sotres-Bayon et al., 2006; Quirk and Mueller, 2008). Therefore, having the mPFC, amygdala, and hippocampus involved in fear extinction allows the adult rat to flexibly express the CS-no-US (i.e., extinc- 
tion) or the CS-US (i.e., conditioning) memory depending on its environment. The data reported here suggest that this network is mature in P24 rats because the mPFC was critical for long-term extinction at this age. Alongside previous findings that extinction in P24 rats is $N$-methyl-D-aspartate (NMDA), GABA, and amygdala dependent and context specific, the present findings indicate that fear extinction at this age is similarly flexible as it is in the adult rat because of the similar neural circuitry. That is, the present results show that extinction training leads to phosphorylation of MAPK in the IL and the amygdala in P24 rats. Previous studies have shown that inhibiting the MAPK pathway in these neural structures significantly impairs extinction in adult rats (Hugues et al., 2004, 2006; Herry et al., 2006). Although it is likely, based on the present findings, that inhibition of the MAPK pathway in these structures will impair extinction learning in P24 rats, future research is required to definitively address that issue. It should also be noted that caution should be taken in interpreting the pMAPK data in the PrL of P24 extinguished rats in this study. That is, these data were not statistically significant but the pattern of results was strikingly similar to that observed in the IL, only with much more variability. Future research will need to examine the exact role of the PrL in extinction.

Overall, the results show that the mPFC is not a part of the neural circuitry underlying fear extinction in P17 rats. As a consequence, we propose that extinction in P17 rats relies on an inflexible system that does not allow for the expression of a previously learned fear once it has been extinguished. In other words, extinction may be unlearning at this age, at least in a functional sense. This idea is consistent with previous findings that show extinction in P17 rats may involve erasure because extinguished fear does not return after changes in the context, pretest reminders, or a pretest reduction in GABAergic inhibition. Further evidence for extinction as erasure in P17 rats comes from a report that pre-extinction systemic injection of the NMDA antagonist MK-801 does not disrupt extinction in 17-d-old rats (Langton et al., 2007; J. H. Kim and R. Richardson, unpublished observations). The NMDA-dependent molecular cascade of fear acquisition and extinction in the adult rat is taken as evidence for the new learning account of extinction (Falls et al., 1992; for review, see Lattal et al., 2006). Hence, NMDAindependent extinction in P17 rats suggests that extinction may not be new learning. Also supporting this position is the recent finding that the amygdala is critical for re-extinction in P17 rats (Kim and Richardson, 2008). In the P24 and adult rat, the amygdala is important only if extinction happens for the first time but appears unimportant for re-extinction (Kim and Richardson, 2008; Laurent et al., 2008). That is, when a fear-eliciting CS was extinguished, reconditioned, and then re-extinguished, rats exhibited low levels of freezing (i.e., good extinction) the next day regardless of whether the amygdala was inactivated or not at the time of re-extinction. From this, it appears that the amygdala is involved in the initial learning of the CS-no-US memory acquired in extinction training, but once that memory has been acquired then the amygdala is no longer needed for subsequent extinction training episodes, at least in postweanling-aged rats. We have shown that re-extinction is amygdala dependent if initial extinction occurs at $17 \mathrm{~d}$ of age and amygdala independent if initial extinction occurred at $24 \mathrm{~d}$ of age, even when training, retraining, re-extinction, and test age were kept constant. This finding suggests that extinction involves unlearning of the original CS-US association in P17 rats; thus, subsequent re-extinction still requires the amygdala. Interestingly, a recent study demonstrated that endogenous opioids are necessary for extinction acqui- sition in P17 rats, as has been demonstrated in P24 rats (Kim and Richardson, 2009) and adult rats (e.g., McNally and Westbrook, 2003). Therefore, a potential neural mechanism for extinction in P17 rats may be opioid-mediated erasure of conditioned fear (for more detail, see Kim and Richardson, 2009) or opioid-induced inhibition of the amygdala (Faber and Sah, 2004); however, it is clear that more studies are necessary to delineate the processes underlying extinction early in development.

We have shown that extinction of learned fear in preweanling rats depends on a fundamentally different neural circuitry compared with older rats. Current neural models of extinction do not predict this developmental dissociation; therefore, further research in this area appears necessary, considering the potential clinical implications of this finding. Specifically, anxiety disorders are thought to originate from traumatic memories from infancy (e.g., Jacobs and Nadel, 1999). However, the present findings suggest that because of the qualitatively different neural processes underlying extinction across development, fear acquired early in life can be much more effectively extinguished if treated early in life. Numerous recent reviews on extinction now suggest both "new learning" and "erasure" as mechanisms for extinction (Delamater, 2004; Barad et al., 2006; Lattal et al., 2006; Myers and Davis, 2007). One of the reasons for this reintroduction of "unlearning" as a potential mechanism for extinction is that recovery of extinguished fear (e.g., renewal, spontaneous recovery) is seldom complete. Another reason is that extinction leads to permanent reductions in synaptic potentials in some amygdala neurons that expressed increased synaptic potentials as a result of previous conditioning (for review, see Barad et al., 2006). Therefore, the dominant mechanism for extinction may be new learning, but that does not rule out erasure as a possible mechanism mediating extinction in the adult rat (Lattal and Stafford, 2008). This balance between erasure and new learning may be simply shifted when extinction occurs early in development compared with when it occurs in adulthood, such that extinction relies more on erasure than on new learning.

\section{References}

Barad M, Gean PW, Lutz B (2006) The role of the amygdala in the extinction of conditioned fear. Biol Psychiatry 60:322-328.

Bouton ME (2002) Context, ambiguity, and unlearning: sources of relapse after behavioral extinction. Biol Psychiatry 52:976-986.

Condé F, Maire Lepoivre E, Audinat E, Crépel F (1995) Afferent connections of the medial frontal cortex of the rat. II. Cortical and subcortical afferents. J Comp Neurol 352:567-593.

Davis M, Myers KM (2002) The role of glutamate and gamma-aminobutyric acid in fear extinction: clinical implications for exposure therapy. Biol Psychiatry 52:998-1007.

Delamater AR (2004) Experimental extinction in Pavlovian conditioning: behavioral and neuroscience perspectives. Q J Exp Psychol 57:97-132.

Faber ES, Sah P (2004) Opioids inhibit lateral amygdala pyramidal neurons by enhancing a dendritic potassium current. J Neurosci 24:3031-3039.

Falls WA, Miserendino MJ, Davis M (1992) Extinction of fear-potentiated startle: blockade by infusion of an NMDA antagonist into the amygdala. J Neurosci 12:854-863.

Fanselow MS (1980) Conditioned and unconditional components of postshock freezing. Pavlov J Biol Sci 15:177-182.

Harris JA, Westbrook RF (1998) Evidence that GABA transmission mediates context-specific extinction of learned fear. Psychopharmacolgy 140:105-115.

Herry C, Trifilieff P, Micheau J, Lüthi A, Mons N (2006) Extinction of auditory fear conditioning requires MAPK/ERK activation in the basolateral amygdala. Eur J Neurosci 24:261-269.

Hobin JA, Goosens KA, Maren S (2003) Context-dependent neuronal activity in the lateral amygdala represents fear memories after extinction. J Neurosci 23:8410-8416.

Hugues S, Deschaux O, Garcia R (2004) Postextinction infusion of a 
mitogen-activated protein kinase inhibitor into the medial prefrontal cortex impairs memory of the extinction of conditioned fear. Learn Mem 11:540-543.

Hugues S, Chessel A, Lena I, Marsault R, Garcia R (2006) Prefrontal infusion of PD098059 immediately after fear extinction training blocks extinction-associated prefrontal synaptic plasticity and decreases prefrontal ERK2 phosphorylation. Synapse 60:280-287.

Jacobs WJ, Nadel L (1999) The first panic attack: a neurobiological theory. Can J Exp Psychol 53:92-107.

Kandel ER (2001) The molecular biology of memory storage: a dialogue between genes and synapses. Science 294:1030-1038.

Kim JH, Richardson R (2007a) A developmental dissociation of context and GABA effects on extinguished fear in rats. Behav Neurosci 121:131-139.

Kim JH, Richardson R (2007b) A developmental dissociation in reinstatement of an extinguished fear response in rats. Neurobiol Lean Mem 88:48-57.

Kim JH, Richardson R (2008) The effect of temporary amygdala inactivation on extinction and re-extinction of fear in the developing rat: unlearning as a potential mechanism for extinction early in development. J Neurosci 28:1282-1290.

Kim JH, Richardson R (2009) The effect of the $\mu$-opioid receptor antagonist naloxone on extinction of conditioned fear in the developing rat. Learn Mem 16:161-166.

Langton JM, Kim JH, Nicholas J, Richardson R (2007) The effect of NMDAreceptor antagonist MK801 on the acquisition and extinction of learned fear in the developing rat. Learn Mem 14:665-668.

Lattal KM, Stafford JM (2008) What does it take to demonstrate memory erasure? Theoretical comment on Norrholm et al. (2008). Behav Neurosci 122:1186-1190.

Lattal KM, Radulovic J, Lukowiak K (2006) Extinction: does it or doesn't it? The requirement of altered gene activity and new protein synthesis. Biol Psychiatry 60:344-351.

Laurent V, Marchand AR, Westbrook RF (2008) The basolateral amygdala is necessary for learning but not relearning extinction of context conditioned fear. Learn Mem 15:304-314.

Likhtik E, Popa D, Apergis-Schoute J, Fidacaro GA, Paré D (2008) Amygdala intercalated neurons are required for expression of fear extinction. Nature 454:642-645.

McDonald AJ (1985) Immunohistochemical identification of $\gamma$-aminobutyric acid-containing neurons in the rat basolateral amygdala. Neurosci Lett 53:203-207.
McNally GP, Westbrook RF (2003) Opioid receptors regulate the extinction of Pavlovian fear conditioning. Behav Neurosci 117:1292-1301.

Milad MR, Quirk GJ (2002) Neurons in medial prefrontal cortex signal memory for fear extinction. Nature 420:70-74.

Millhouse OE (1986) The intercalated cells of the amygdala. J Comp Neurol 247:246-271.

Mineka S, Zinbarg R (2006) A contemporary learning theory perspective on the etiology of anxiety disorders. Am Psychol 61:10-26.

Morgan MA, Schulkin J, LeDoux JE (2003) Ventral medial prefrontal cortex and emotional perseveration: the memory for prior extinction training. Behav Brain Res 146:121-130.

Myers KM, Davis M (2007) Mechanisms of fear extinction. Mol Psychiatry 12:120-150.

Newman DL, Moffitt TE, Caspi A, Magdol L, Silva PA, Stanton WR (1996) Psychiatric disorder in a birth cohort of young adults: prevalence, comorbidity, clinical significance, and new case incidence from ages 11 to 21 . J Consult Clin Psychol 64:552-562.

Pavlov IP (1927) Conditioned reflexes. London: Oxford UP.

Paxinos G, Watson C (1998) The rat brain in stereotaxic coordinates. New York: Academic Press.

Pine DS, Helfinstein SM, Bar-Haim Y, Nelson E, Fox NA (2009) Challenges in developing novel treatments for childhood disorders: lessons from research on anxiety. Neuropsychopharmacology 34:213-228.

Quirk GJ, Mueller D (2008) Neural mechanisms of extinction learning and retrieval. Neuropsychopharmacology 33:56-72.

Sesack SR, Deutch AY, Roth RH, Bunney BS (1989) Topographical organization of the efferent projections of the medial prefrontal cortex in the rat: an anterograde tract-tracing study with Phaseolus vulgaris leucoagglutinin. J Comp Neurol 290:213-242.

Sierra-Mercado D Jr, Corcoran KA, Lebrón-Milad K, Quirk GJ (2006) Inactivation of the ventromedial prefrontal cortex reduces expression of conditioned fear and impairs subsequent recall of extinction. Eur J Neurosci 24:1751-1758.

Sotres-Bayon F, Cain CK, LeDoux JE (2006) Brain mechanisms of fear extinction: historical perspectives on the contribution of prefrontal cortex. Biol Psychiatry 60:329-336.

Weber M, Richardson R (2001) Centrally-administered corticotropin-releasing factor and peripheral injections of strychnine hydrochloride potentiate the acoustic startle response in preweanling rats. Behav Neurosci 115:1273-1282. 